University of Vermont

UVM ScholarWorks

University Libraries Faculty and Staff

Publications

University Libraries

2017

\title{
Guide on the Side and LibWizard Tutorials side-by-side: How do the two platforms for split-screen online tutorials compare?
}

\author{
Graham Sherriff \\ University of Vermont, graham.sherriff@uvm.edu
}

Follow this and additional works at: https://scholarworks.uvm.edu/libfacpub

Part of the Information Literacy Commons

\section{Recommended Citation}

Sherriff, Graham, "Guide on the Side and LibWizard Tutorials side-by-side: How do the two platforms for split-screen online tutorials compare?" (2017). University Libraries Faculty and Staff Publications. 46. https://scholarworks.uvm.edu/libfacpub/46

This Article is brought to you for free and open access by the University Libraries at UVM ScholarWorks. It has been accepted for inclusion in University Libraries Faculty and Staff Publications by an authorized administrator of UVM ScholarWorks. For more information, please contact scholarworks@uvm.edu. 


\title{
TITLE: Guide on the Side and LibWizard Tutorials side-by-side: How do the two platforms for split-screen online tutorials compare?
}

Author:

Graham Sherriff, Instructional Design Librarian, University of Vermont, Burlington, Vermont, USA

Received 08 Dec 2016, Accepted 12 Feb 2017.

Address correspondence to Graham Sherriff, Bailey/Howe Library, University of Vermont, Burlington, VT 05405. E-mail: graham.sherriff@uvm.edu

This is a post-print Author's Accepted Manuscript of an Article published in the Journal of Web Librarianship, March 24, 2017, available online at:

http://dx.doi.org/10.1080/19322909.2017.1295821

\begin{abstract}
Split-screen tutorials are an appealing and effective way for libraries to create online learning objects where learners interact with real-time web content. Many libraries are using the University of Arizona's award-winning, open source platform, Guide on the Side; in 2016, Springshare released a proprietary alternative, LibWizard Tutorials. This article reviews the advantages and limitations of this kind of tutorial. It also examines the differences between each platform's distinctive characteristics. These platforms create similar split-screen tutorials, but have differences that affect diverse aspects of installation, administration, authoring and editing, student learning, data management, and accessibility. Libraries now have the opportunity to
\end{abstract}


consider and compare alternative platforms and decide which one is best suited to their needs, priorities and resources.

\section{Keywords}

split-screen tutorials, digital learning objects, web services, flipped instruction, online learning, learner performance data, information literacy

\section{Introduction}

The options for producing online tutorials are proliferating rapidly as online, distance, and hybrid instruction expands across higher education. Increased options are helpful for libraries that are flipping instruction, embedding in learning management systems (LMS), or creating their own online courses, although keeping up with new products is a constant challenge. One model adopted by some college and university libraries in recent years is the split-screen, live-web tutorial. These tutorials are a type of learning object that engage learners with real-time web content. The learner accesses the tutorial via a URL, perhaps posted in their course's LMS shell or on the library website. This URL routes to the web-hosted tutorial, which divides the screen

into two frames. ${ }^{1}$ A narrow left-side frame displays authored content (which this article refers to as the "tutorial frame"). A larger right-side frame displays live-web content that the learner can view and interact with (the "web frame"). See Figure 1. 


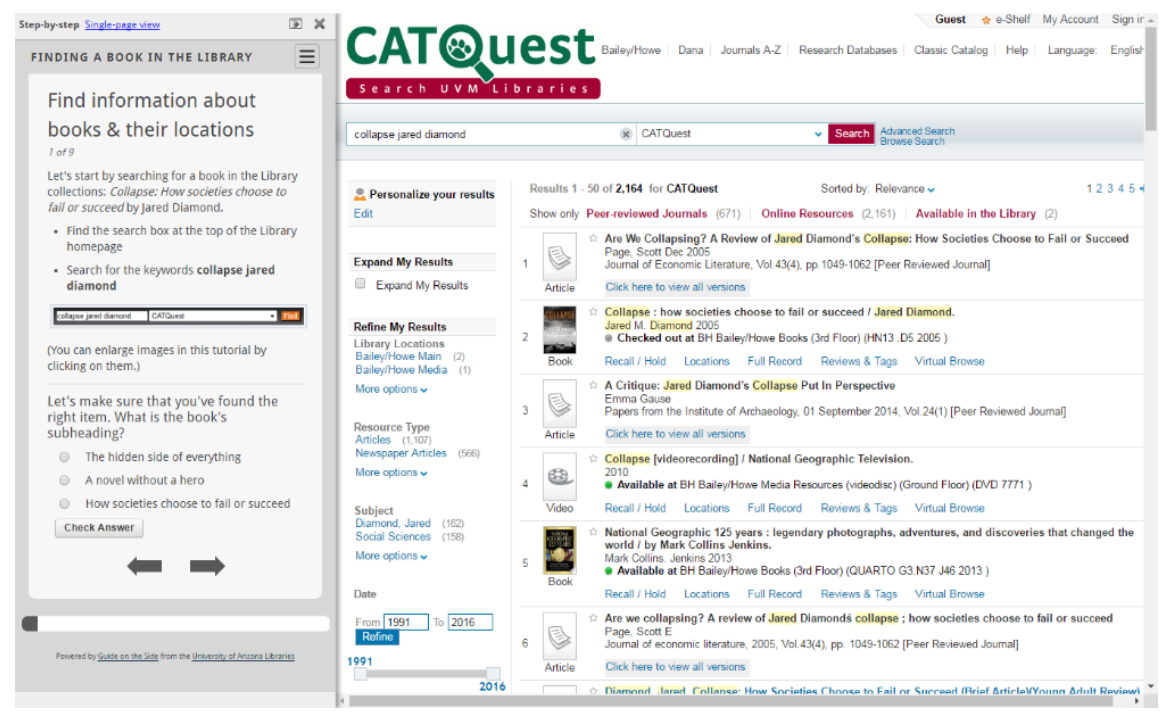

FIGURE 1. The learner interface in a split-screen, live-web tutorial (Guide on the Side).

Typically, the tutorial frame presents information, gives directions for tasks the learner needs to complete in the web frame, and poses questions the learner needs to answer. This frame is also where the learner submits responses to those questions. The web frame allows the learner to engage autonomously and dynamically with the web by scanning, scrolling, navigating, and searching in order to complete the tasks and develop responses to the questions. For example, a tutorial might direct the learner to navigate to an online resource, enter a search query, and answer questions that require them to reflect on their results. The learner controls the pace of their progression through the tutorial and can be allowed to skip backwards and forwards, repeating or previewing sections as they like.

The integration of live-web content has substantial benefits for learning:

- The learner's interaction with web content gives the learner the autonomy to make their own decisions about how to execute directions, complete tasks, or respond to what they 
are experiencing. This freedom of choice has the potential to support a constructivist approach to instructional design, which suggests that learning results from the learner's activity, experiences, and interpretations. In addition, as Stonebraker notes, it gives the learner hands-on practice in activities that they will subsequently need to perform independently (2015).

- The learner engages with the actual, real-time, fully featured web, making it a richer, more dynamic and more authentic experience than captured content like screencasts, slides, or storyboards. Including authentic activities also has the potential to support a constructivist approach, which guides the learner towards the development of knowledge through their engagement with their external environment.

- The learner has access to an (almost) web-scale range of content, activity and decisionmaking. A single tutorial might span the library website, licensed resources, open educational resources, webpages, blog posts, and other web-hosted material.

The incorporation of live-web content also has advantages for tutorial creators. Composition is mostly text editing and does not require the time and expertise required to compose images, animation, or scripts. It also reduces the amount of maintenance by eliminating much of the need to update and republish a tutorial every time a webpage redesigns its layout or labelling.

However, the use of frames to present the web content presents some limitations:

- There is no audio narrative, which may make tutorials less effective for students with a primarily auditory learning style. 
- Some online content is not available. Some sites configure the $\mathrm{x}$-frame-options in their source code to prevent third-party sites from embedding their content. ${ }^{2}$ Frustratingly, this includes several high-profile websites that librarians might want to feature in their tutorials. These websites include Google, Google Scholar, Google Patents, Google News, the New York Times, Twitter, and Flickr. There have also been reports of problems with live-web content from PubMed and RefWorks (Schmidt and Hartman 2015).

- Learners are likely to be unfamiliar with the split-screen model and may need instruction in how the screen is structured and how to engage with each frame.

In the 2000s, a small number of libraries experimented with homegrown versions of this model independently of each other (Sult et al. 2013). The first program made available for adoption by other libraries was Guide on the Side (GotS), an open source platform developed and maintained by the University of Arizona Libraries, released in July 2012. In February 2016, Springshare LLC released an alternative product when it added a "Tutorials \& Assessments" module (henceforth simply "LibWizard Tutorials") to its proprietary LibWizard platform (Pawlek 2016a).

LibWizard Tutorials and GotS are comparable platforms for the creation of split-screen tutorials. They use the same split-screen structure based on dual frames, they both integrate live-web content, and they have similar quizzing features. See Figure 2. 


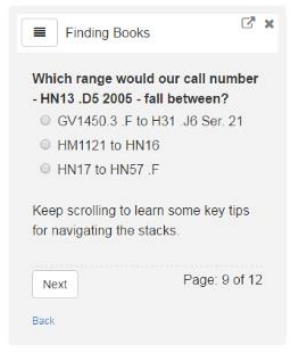

You will find signs at the end of each row of books. These signs tell you the call number range in that row.

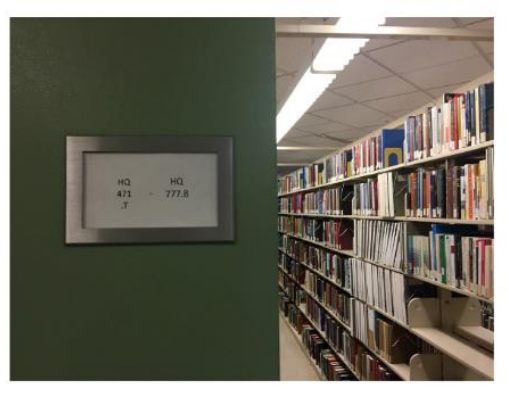

FIGURE 2. The learner interface in a LibWizard tutorial.

However, many of their features have different characteristics and behaviors, and each has unique features that are not available in the other. Consequently, there are differences between the two with significant implications for implementation, administration, authoring and editing, usability and the learner experience, accessibility, instructional use, and assessment. These differences give librarians an opportunity to compare alternative programs and evaluate how suited each one is to their institution's priorities and circumstances.

\section{Literature Review}

Unsurprisingly, given LibWizard's short history, the literature on this type of online tutorial is almost entirely limited to studies of GotS. The program originated at the University of Arizona, where librarians wanted tutorials to support increased demand from faculty for library instruction, but with more interactivity and authenticity of experience than screencast videos could offer (Bracke and Dickstein 2002). Following implementation in 2010, the University of 
Arizona's tutorials generated "remarkably positive" feedback from faculty, instructors, and students (Sult et al. 2013).

Studies have demonstrated that appropriately designed GotS tutorials have a quantifiable and positive effect on learner performance (Mery et al. 2014) and an effect size greater than that of equivalent screencast tutorials (Stonebraker et al. 2016). But more studies on learning effects are needed in order to substantiate these findings and test whether they can be generalized.

What is "appropriate design" for a frame-based, live-web tutorial? Best practices proposed by librarians who worked on implementation at the University of Arizona include:

- Selecting an appropriate starting point;

- Giving the learner multiple opportunities to interact with the web content;

- Using questions to ensure students are viewing the correct content;

- Using questions to check comprehension;

- Using styling and layout to emphasize important words in the tutorial frame;

- Making directions as clear as possible; and

- Reviewing tutorials regularly (Mery et al. 2013).

Looking at the production side of GotS, the time needed to create and maintain split-screen tutorials may be significantly longer than expected. The authors of the first GotS tutorial estimated that development took 70 hours $^{3}$ and student support during the assignment needed another 20 hours (Bracke and Dickstein 2002). Compared with the production time for an 
equivalent screencast, production may take more than three times as long (Mikkelsen and McMunn-Tetangco 2014). The latter study suggests the quizzing component may be a significant time-cost.

Another time-cost may be the extended reworking that is possible in GotS. It is possible to make very small edits to text, layout, and questions, which may encourage continuous revisions and adjustments. In contrast, screencasts typically comprise lengthy captures of audio or video, such that the time needed to make a revision is a disincentive to making minor or non-essential edits.

Another way in which the profession has communicated the positive impact of GotS on library instruction is through peer recognition. University of Arizona developers Mike Hagedon and Leslie Sult received the Association of College and Research Libraries (ACRL) Instruction Section's 2013 Innovation Award in recognition of their work on the platform. There has also been recognition for individual GotS tutorials. The ACRL Instruction Section has accepted two GotS-based tutorials into its Peer-Reviewed Instructional Materials Online Database (PRIMO): the University of Vermont's tutorial “Evaluating Information” (DeFrain 2013) and the University of South Carolina’s tutorial “Search for History Books” (Snediker 2015).

Springshare released LibWizard Tutorials in early 2016 and therefore it has not yet received the same scrutiny or assessment as GotS. The Charleston Adviser's product review provides a detailed explanation of the authoring layout and features, and describes both the back-end interface and the learner interface as "simple and easy to use" (Kaletski 2016, 23). However, the product review examines the whole LibWizard product, in which Tutorials is one of four 
modules. As a result, the comparison of LibWizard with other products provided in the Adviser review weighs it against Google Forms but not in comparison to GotS or any other tutorial platform.

\section{Context}

This evaluation was motivated by the University of Vermont (UVM) Libraries' need to consider the suitability of each tutorial platform. The University of Vermont is a medium-sized, public, doctoral research university that has used GotS since 2013 and is piloting LibWizard Tutorials in the 2016-17 academic year to consider the desirability and feasibility of a switch in platforms. The UVM Libraries adopted GotS principally to flip some of the information literacy instruction that it has integrated into courses for a university-level Foundational Writing and Information Literacy (FWIL) initiative. All freshmen take a course within this initiative. Each course has a research component, supported by a one-shot library session.

The primary objective for this flipped instruction is to provide all freshmen with instruction in key information literacy concepts and basic research skills. Students complete five online tutorials in advance of the one-shot. ${ }^{4}$ Flipping instruction in this way establishes a baseline knowledge and enables instruction librarians to engage with specific questions and difficulties that students are experiencing, when meeting with FWIL sections in the classroom.

The UVM Libraries selected GotS for these tutorials on the basis that it met three key criteria: 
- Frame-based, live-web tutorials are a powerful learning tool. As well as the beneficial features that are general to this model of tutorial, described above, GotS has features that make it well suited to a formative learning experience. This instructional design choice is particularly important in the context of our FWIL courses and the need to bring students to a common level of knowledge and understanding, as opposed to using tutorials for assessment.

- GotS tutorials (like LibWizard Tutorials) are highly scalable. The UVM Libraries' highest-use tutorials are completed by around 2,500 students every year.

- GotS is open access and therefore free in financial terms, while the library's Systems department was willing to install the program and provide back-end technical support.

Since adoption in 2013, UVM librarians have extended our use of GotS to meet other needs for online learning objects:

- A suite of beginner's-level tutorials covering research basics, hosted on the library website for "off-the-shelf" access;

- Discipline-specific tutorials for other courses, at both foundational and advanced levels;

- Orientation-style guides to library services; and

- In-house training.

\section{Method}


When Springshare released LibWizard Tutorials in 2016, the UVM Libraries arranged a trial and found some of its unique features impressive. At the same time, we noted the absence of some GotS features. We wanted to give full consideration to the two platforms' relative merits, and also the significance of their differences. We therefore designed a comparative evaluation, using a rubric based on the UVM Libraries' instructional priorities and resources for platform administration.

Our rubric identified 22 performance standards that were important for our local circumstances. For example, “Tutorials allow the learner to make decisions and submit responses without penalty;" and "Adoption and use by instructional librarians with low-to-moderate technical complexity."

Each standard was classed as "Essential" (integral to how our tutorials work and the attainment of learning outcomes); "Important" (elements that are broadly desirable but not essential); or "Optional" (elements that offer minor enhancements).

A working group of the UVM Libraries' tutorial administrators and main users then evaluated the extent to which GotS and LibWizard Tutorials met the defined standards. They did this by applying the rubric to live tutorials in GotS and test tutorials in LibWizard Tutorials, and on the basis of group discussions.

\section{Versions}


The following evaluation of GotS and LibWizard Tutorials is less specific to local needs. Instead, it tries to present a neutral evaluation that may be useful to a broad range of libraries. It describes GotS and LibWizard Tutorials as they were in November 2016: GotS at version 1.0beta4 (mhagedon 2016) and LibWizard Tutorials at version 1.7.6 (Pawlek 2016b). Both platforms have undergone several updates since their launch and further updates are likely, especially for LibWizard Tutorials, given Springshare's customary practice of continuous development and frequent updating.

The University of Arizona Libraries announce GotS updates via the "Guide on the Side News" feed on the libraries' website (http://code.library.arizona.edu/) and the Google Group “Guide on the Side discussion." Springshare announces LibWizard updates via its blog (http://blog.springshare.com/).

\section{Installation}

GotS is an open source program, available for downloading from the University of Arizona Libraries website or via cloning from GitHub. It is written in the CakePHP framework, designed to run on a Linux platform, and requires a MySQL database and email configuration (caosborne89 et al. 2016). In other words, technical expertise is required. "To install the application at your institution, you need the skills, access and resources that are common to installing a web application" (University of Arizona Libraries 2015).

LibWizard is a proprietary, cloud-based program, accessed through Springshare's LibApps platform of library-oriented web applications. As noted, Tutorials is one of four modules in 
LibWizard. Two of the LibWizard modules are included in Springshare's LibApps CMS license as "LibWizard Lite," but LibWizard Tutorials (and Quizzes) requires a separate license. Pricing depends on library size, specifically FTE for academic libraries; Kaletski (2016) provides greater detail about licensing terms. LibWizard subscribers can create two types of tutorial:

"Standalone," the split-screen tutorial model; and "Embedded," a pop-up tutorial accessed via a webpage's floating side-tab (not discussed here).

The difference between local installation and licensing has obvious implications for long-term sustainability. A library can maintain its GotS tutorials indefinitely, while continued use of LibWizard tutorials is tied to an active site license.

\section{Administration}

Both platforms have modest system-level maintenance requirements, mostly relating to basic configurations and styling. The principal difference is that administration of a GotS installation requires access to the program files and the necessary programming skills. LibWizard administrators work entirely through a front-end admin interface, which is accessible to any user assigned "admin" status. This admin interface includes simple text fields for custom CSS or JavaScript, as well as for changes to in-tutorial labels and messages.

In GotS, account holders are "admins" or "users," and the only additional privilege held by admins is access to the "User Management" index, where administrators can create, edit, and 
delete accounts. Anyone with "user" status can access any tutorial, including the ability to edit or delete another user's tutorial.

In LibWizard Tutorials, account holders are "admins" or "regulars." Administrative privileges include access to the site settings and access to all tutorials. Regulars cannot see another user's tutorials or performance data. In one respect, this is a layer of privacy for librarians' work and a measure of confidentiality for learner data. But at the same time, individual ownership and the accompanying restrictions on visibility and sharing may be seen as a barrier to collaboration on tutorial design. There is no option for co-owning or co-editing a tutorial.

Technical support for GotS is limited. The University of Arizona Libraries provide some documentation on their website and maintain the Google Group. Discussion threads in the Google Group are infrequent and mostly raise questions about installation. The GotS developers at the University of Arizona typically respond to questions that are not immediately answered by other members. This level of support may be sufficient for libraries that prefer to keep systems in-house and have personnel available to provide technical support as needed.

Springshare offers a substantial and more robust range of options for technical support, which may be preferable for libraries with fewer resources for the management of web services or libraries that would manage a subscription through a non-technical department. These options include a highly responsive helpdesk, online documentation, training webinars and archived videos, a dedicated LibWizard discussion forum in the Springshare Lounge website 
(http://springsharelounge.com), and Springshare reps serving as a reliable point of contact for each subscribing institution. ${ }^{5}$

Importantly, tutorials are highly scalable in both GotS and LibWizard. Users can access tutorials simultaneously and each platform can process an effectively unlimited volume of performance data.

\section{Authoring and editing}

The interfaces and processes for authoring and editing tutorials in GotS and LibWizard Tutorials are both user-friendly and mastering the available features is more or less straightforward. Also, they are both compatible with Mac and Windows operating systems. But the interfaces and their features have important differences.

GotS uses a WYSIWYG text editor with just 11 features. See Figure 3. Users can add content to the tutorial frame as text, images, links, accordion-style "definition" boxes, or multiple-choice or free-response questions. There is no code view. As noted, the University of Arizona originally developed GotS with a code editor, then replaced this method of editing with a text editor to accommodate the needs of a user base that would typically comprise instruction librarians lacking the coding skills required by a code editor (Sult et al. 2013). 


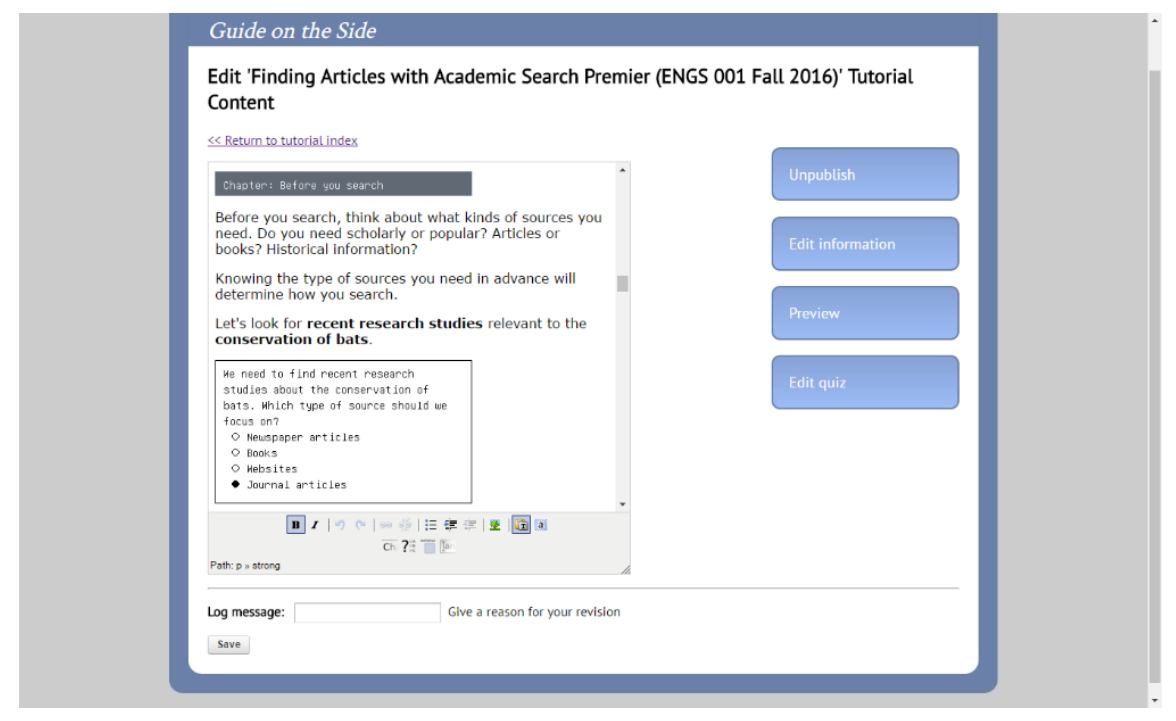

FIGURE 3. The authoring interface in GotS, with a word processing-style text editor and simplified features.

The GotS authoring interface resembles a highly simplified word processing program. This familiar interface makes it usable for librarians of all levels of technological expertise, although some users have criticized the lack of styling features like colored text and adjustable typefaces (Atwood 2015; Schmidt and Hartman 2015). The word-processor style interface also makes it easy to view the tutorial content when scrolling up and down in the text editor, and to copy and paste chunks of text as needed.

In contrast, LibWizard has a segmented authoring model. The interface has a split-screen layout that is similar to the appearance of a LibWizard tutorial. In the larger right-side frame, the author develops the tutorial by assembling a series of slides. Each slide must be separately labeled, assigned content, and positioned appropriately in the tutorial sequence by dragging and dropping. In the left-side frame, the author can adjust the tutorial settings. See Figure 4. 


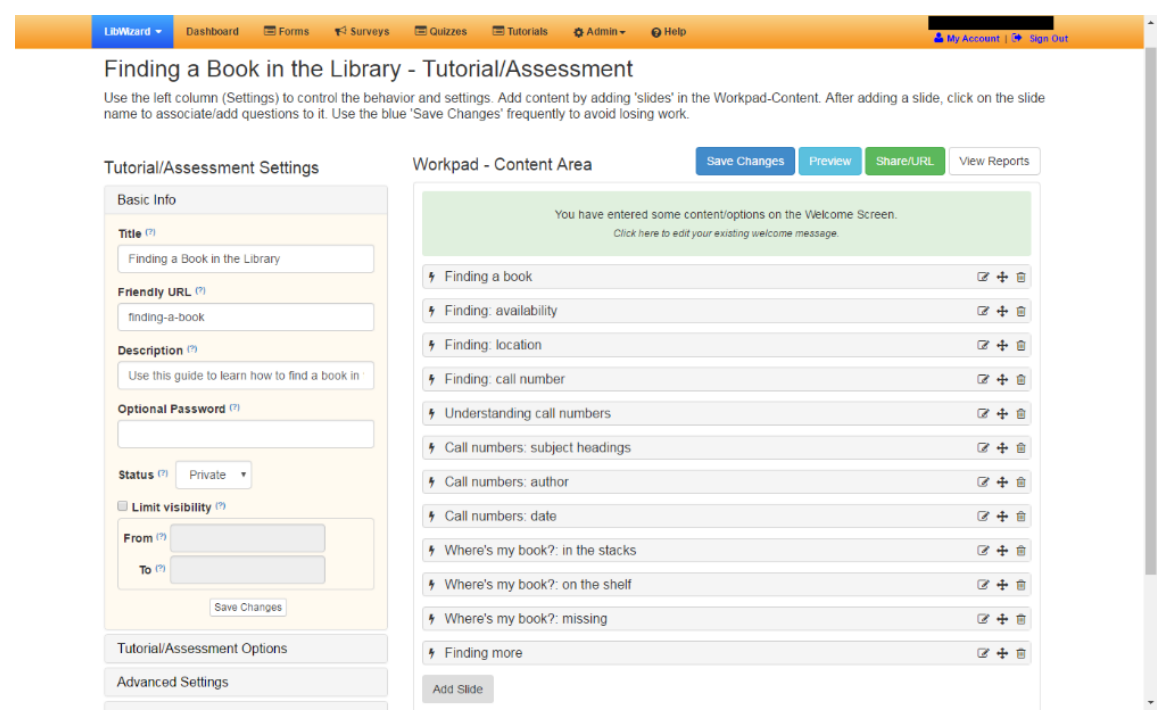

FIGURE 4. The tutorial-level authoring interface in LibWizard Tutorials. Slides are created, labeled, and then positioned appropriately in the "Workpad - Content Area."

For each slide, the author creates tutorial-frame content in a similar way to creation of slides: by selecting discrete content items, labeling them, and positioning them in the tutorial by dragging and dropping. See Figure 5. LibWizard has a larger range of options for tutorial-frame content than GotS. In addition to the options available in GotS, LibWizard's content item types include a 1-to-5 rating scale, a grid, and an input field that will validate the format of submitted data (e.g., “Date \& Time Input”, "Submitter Email”). The user can toggle LibWizard's text field editor between a WYSIWYG view and an HTML view. 


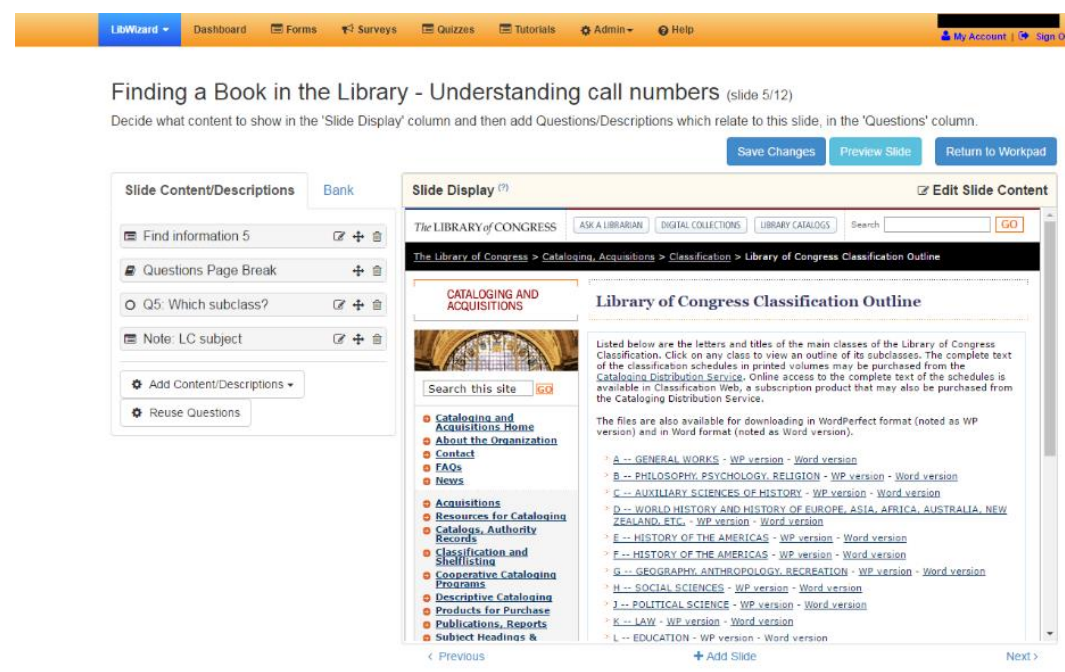

FIGURE 5. The slide-level authoring interface in LibWizard. Content is created with the leftframe "Content/Descriptions" options.

The differences between the word processor-based and segment-based interfaces have significant implications. First, consider how each platform presents tutorial content and how the learner engages with it. GotS presents a webpage that is the tutorial's starting-point, and from there the learner assumes control of their movement through the content. This design choice means content must be accessible through the web. A librarian who wants to include an image file or a text document will first need to post it online.

Starting with a webpage also means the learner can engage with the web frame content (or not) in whatever way they choose. The tutorial frame can present directions and, when necessary, a clickable URL for direct navigation to a different webpage. But following these directions is at the learner's discretion. As a result, the learner has maximum autonomy and agency, and they have the freedom to fail in their execution of the tutorial's directions. This level of autonomy 
may or may not be desirable from the instructor's point of view, depending on the objectives of the individual tutorial.

A LibWizard tutorial can present a richer array of media content. Each slide can display a starting-point webpage, an embedded video, an image file, a PDF, or a composed text block. The use of slides means the tutorial loads content at a series of points compared with GotS's single starting-point. Having more flexibility with starting points may be advantageous if the tutorial needs to present non-web content, or if it is necessary to check mid-tutorial that the learner is in the right place and viewing the right content.

Slides are also LibWizard Tutorials' way to "airlift" the learner out of one webpage and move them to another webpage not linked to the first. In GotS, a link in the tutorial frame can be set with the main frame as its target. In LibWizard, link targets are limited to new pages in the same tab (i.e., displacing the tutorial page) or new tabs or windows (i.e., leaving the tutorial).

One potential downside to the use of slides is the complexity of mapping them to the tutorial's table of contents (ToC). Both GotS and LibWizard tutorials have the optional feature of displaying a ToC "hamburger" icon that identifies a tutorial's different sections and provides a convenient way to skip ahead or jump back and repeat a section. Additional navigational options are valuable; the literature on learning objects has identified meaningful and intuitive wayfinding as an aid to effective learning (Race 2006). GotS defines the ToC sections as "chapter" sections that the author can position wherever they are needed. In LibWizard, ToC sections are defined by the slides. In situations where the sequence of slides does not align with the intended structure 
for the tutorial, it may be necessary to adjust the titles of some slides or even their content, in order to make the ToC an intuitive navigational aid.

A second implication of the differences between the two authoring interfaces concerns the time and effort to compose and revise content. Using the GotS text editor, an author can compose a tutorial in a continuous flow. Designing a LibWizard tutorial entails planning how it will segment the content into different slides - including how those slides will display as sections in the table of contents, as explained above. The author needs to create, label, populate, and position each slide; and then create, label, populate, and position each discrete content item within each slide. Future research could explore if the GotS interface is more time-efficient than LibWizard's interface for a librarian who is creating a new tutorial or adding new tutorial content. However, LibWizard's segmented authoring model has benefits for editing an existing tutorial. It makes it possible to quickly drag and drop slides and tutorial items. It also makes it possible to reuse tutorial items through a searchable "Question Bank" listing all text and question items in all other tutorials. ${ }^{6}$

One of the desirable characteristics of online tutorials is reusability, meaning the ability of others to copy, modify, and republish. Reusability within a library's platform account supports ease of production and sustainability. Reusability by other libraries supports freedom of information and advances in library instruction across the profession.

Both platforms support in-house reusability. In GotS, authors can simply copy any of their library's tutorials, paste it into a new one, and edit as needed. In LibWizard Tutorials, authors 
can select one of their own tutorials to use as a starting point for creating new tutorials, or any tutorial owned by someone else in their library that has been set to have "Public" status. Tutorials with "Private" status are not visible or replicable across individual accounts.

However, neither platform supports external reusability. Libraries can extend access to the learner-view of their GotS and LibWizard tutorials as widely as they like, for example by posting URLs publicly and waiving intellectual property rights. But neither platform has functionality that would enable a platform user to import another library's tutorial and adapt, rebrand, or update it. Similarly, neither platform has a feature for duplicating another library's tutorial or extending cross-library access.

\section{Formative learning}

As described above, the UVM Libraries adopted GotS for several reasons including its suitability to formative library instruction. The principal objective was to ensure that FWIL students came to the library one-shots having already covered key concepts and technical knowledge in the tutorials, so the instruction librarian could focus on challenges and troublesome questions that arose as students begin to engage with their research.

GotS has several features that support formative learning. Learners can control pacing and progression through the tutorial, as was previously noted. Importantly, GotS can give affirmative feedback that acknowledges the learner's correct responses, reinforces what is being taught, and 
provides motivation to continue. Affirmative feedback was not a feature of LibWizard Tutorials in its initial release, but was a new feature in version 1.7.6.

GotS can also give targeted corrective feedback to incorrect responses. Consequently, feedback is specific to the exact error and explains how the learner should reconsider the question depending on the mistake they made. A LibWizard tutorial can present corrective feedback, but the interface only allows a single statement that is uniform for all of a question's incorrect response options.

Another significant difference lies in the platform's calculations of the tutorial score. GotS calculates the learner's last-submitted responses. As a result, both librarian and student can see whether the learner succeeded in reaching the required point of understanding, regardless of how many efforts they took to get there. LibWizard calculates the learner's first-submitted responses, which only allows the learner one opportunity to be evaluated based on what they learned in the tutorial.

\section{Summative assessment}

The calculation of first-submitted responses is better suited to a summative assessment that tests what the learner knows when they engage with a question for the first time. LibWizard is configured to collect summative assessment. GotS calculates last-submitted responses to questions embedded in the main tutorial - but it is also possible to attach a "quiz" at the end that 
calculates first-submitted responses. Thus GotS tutorials can combine formative and summative assessment in a single tutorial.

\section{Data management}

Both platforms are excellent tools for quizzing learners, but they have strikingly different features for routing, aggregating, and reporting the data generated by learners' responses.

GotS tutorials can email an HTML "certificate" to pre-selected addresses (for example, to the librarian, the course instructor, or a GotS admin account) as well as to addresses entered by the learner (for example, to their own email address or their TA's email). The certificate records a learner's correct responses, incorrect responses, free responses, overall scores, and a time-stamp. See Figure 6. But GotS has no built-in process for aggregating this data. Aggregation requires time-consuming and tedious manual data entry, with a risk of significant error. In the experience of the UVM Libraries and other libraries using GotS, the lack of an automated workflow for data management limits assessment to small-scale sampling; however, applying a script for data aggregation may be feasible for libraries with programming resources. 

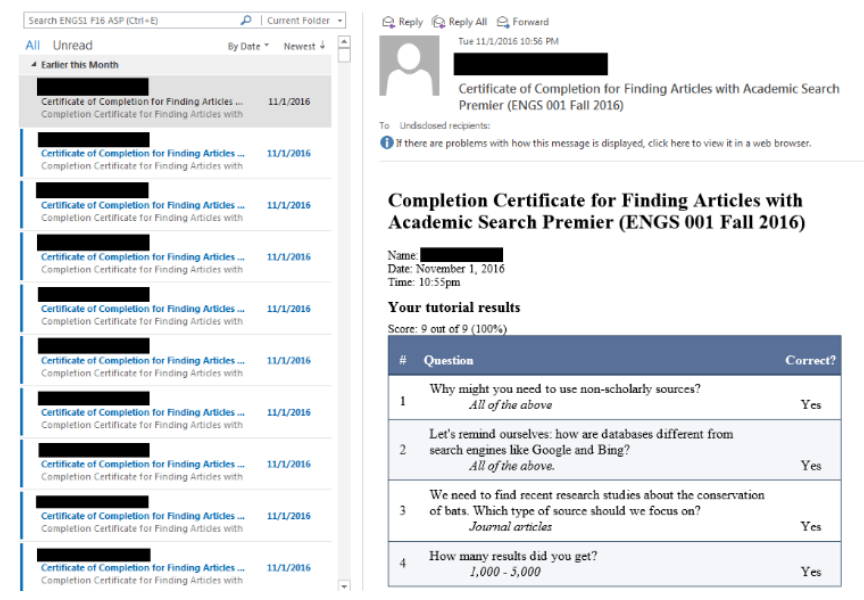

FIGURE 6. A GotS administrator's email account, displaying a typical tutorial certificate. GotS delivers certificates to the learner and other recipients as HTML-based email messages.

As in GotS, a LibWizard tutorial can email a record of the learner's performance to a pre-set address and to addresses submitted by the learner within the tutorial. But LibWizard also offers a range of valuable data management features that facilitate large-scale analysis and assessment. First, it aggregates learner data automatically. The report includes the same data points as in GotS, but with the optional additions of browser, referring URL, and IP address. Second, a tutorial owner or platform administrator can view total data as a table, and data for individual questions as tables or charts. Third, LibWizard can cross-tabulate combinations of questions. Finally, LibWizard can export learner data as CSV files. See Figure 7. 


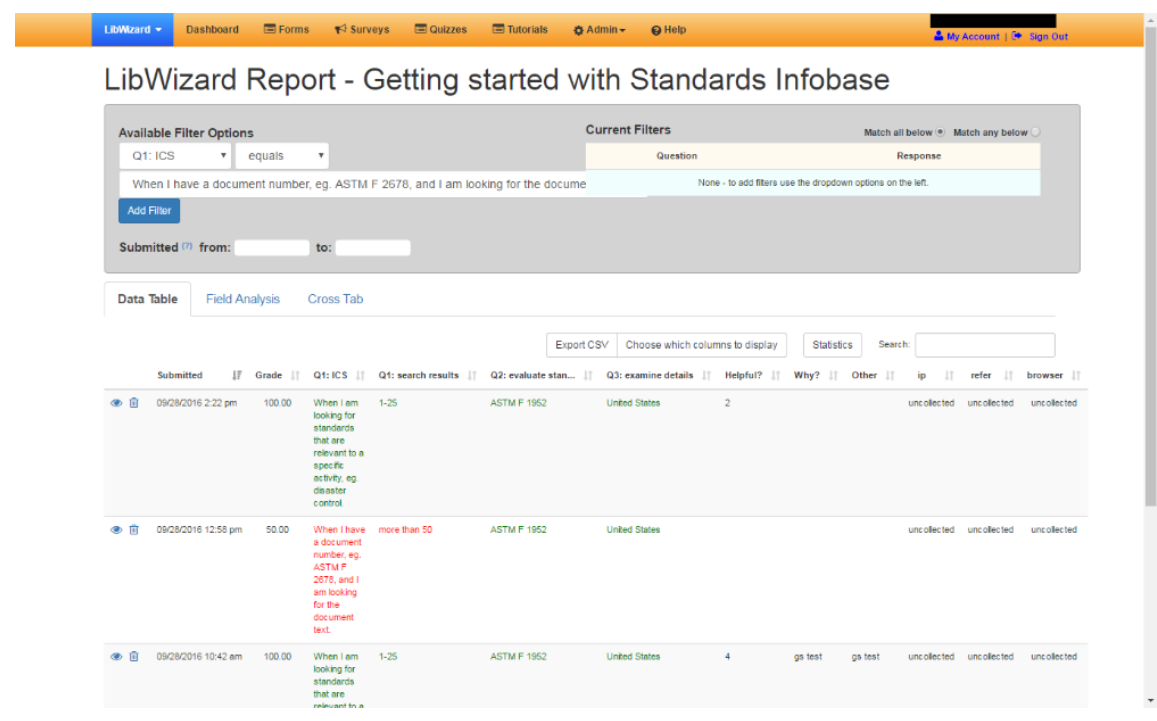

FIGURE 7. The data management interface for a LibWizard tutorial. The default display is a complete and searchable data table, with an option to export the data as a CSV file. Tabs provide access to table and chart views for individual questions, and cross-tabulations of questions.

Some librarians may have reservations about the confidentiality of learner data in LibWizard. As noted, a tutorial's data is visible within the module not just to the tutorial owner, but also to the library's LibWizard admins and - if the tutorial has "Public" status - to all of the library's other LibWizard users. The data is also accessible to Springshare employees and anyone with access to the Springshare server that hosts it. The range of people with access may have implications for what data a librarian may choose to gather with a LibWizard tutorial and whether that data should be personally identifiable. Academic libraries may also need to check whether the platform, or specific uses of the platform, is compatible with institution-level policies on the gathering, ownership, and retention of student data. 


\section{Accessibility}

Compliance with accessibility standards is essential for online instructional materials. Ethically, librarians value the importance of providing equal access to resources and services to all potential library users. Legally, institutions that receive federal funding are required to comply with the accessibility standards in Section 508 of the 1973 Rehabilitation Act.

There is disagreement about the accessibility of split-screen tutorials. Stonebraker et al. report they "are not ideal in terms of accessibility for those patrons using screen readers" because this kind of assistive technology cannot interact with frames $(2015,235)$. However, in their Voluntary Product Evaluation Template (VPAT), a document that self-evaluates levels of compliance with Section 508 for LibWizard, Springshare reports that its learner interface is Section 508-compliant, including compatibility with screen reading software. ${ }^{7}$ As well as compatibility with screen readers, Springshare's VPAT notes other accessibility features: the learner can navigate with a keyboard, mouse, or other hardware; creators can customize the CSS code governing styling such as color contrast; and there are fields for defining link titles and "alt" tags. The VPAT assesses the creator/administration interface as not fully compliant in some areas, due to unspecified complexities (Pawlek n.d.).

GotS does not have a formal VPAT, but it addresses accessibility issues with a "single-page view" that presents the full tutorial content in a single webpage, accessed via a link at the top of the tutorial frame. The University of Arizona designed this one-page version of the tutorial to be compatible with screen readers and keyboard users, and the more accessible version contains the 
same content and the same answer-response quizzing functionality as the dual-frame version (simpsonw and michaelhagedon 2008).

GotS also has some of the accessibility features that are available in LibWizard Tutorials, such as link titles and "alt" tags, and some unique features, such as the option to magnify any image uploaded into the tutorial frame. Administrators with access to the installation files can customize the CSS stylesheet, though changes can only be applied site-wide.

\section{Conclusions and Future Developments}

Sometimes the devil is in the details. At first glance, a GotS tutorial and a LibWizard tutorial may appear very similar. But the differences in their platform features manifest themselves in a variety of ways, with significant impacts on administration, authoring, and the learner experience. For a comprehensive overview of these features, see Table 1.

\begin{tabular}{|l|l|l|}
\hline Feature & Guide on the Side 1.0-beta4 & LibWizard Tutorials 1.7.6 \\
\hline \hline Procurement & $\begin{array}{l}\text { - Open source; available as a } \\
\text { free download }\end{array}$ & $\begin{array}{l}\bullet \text { Proprietary; available as a paid } \\
\text { license }\end{array}$ \\
\hline $\begin{array}{l}\text { Installation \& back- } \\
\text { end maintenance }\end{array}$ & $\begin{array}{l}\text { - Must be installed on a local } \\
\text { server } \\
\text { Requires programming } \\
\text { expertise }\end{array}$ & $\begin{array}{l}\bullet \text { Cloud-based } \\
\text { Technical expertise not } \\
\text { required }\end{array}$ \\
\hline Technical support & $\begin{array}{l}\text { Informal, relies on user } \\
\text { community }\end{array}$ & $\begin{array}{l}\text { Vendor provides support desk } \\
\text { and extensive documentation }\end{array}$ \\
\hline $\begin{array}{l}\text { Authoring } \\
\text { interfaces }\end{array}$ & $\begin{array}{l}\text { Simple WYSIWYG text } \\
\text { editor }\end{array}$ & $\begin{array}{l}\text { Author composes slides, then } \\
\text { composes content items for } \\
\text { each slide } \\
\text { WYSIWYG and code editors }\end{array}$ \\
\hline
\end{tabular}




\begin{tabular}{|c|c|c|}
\hline Media content & - Webpages & $\begin{array}{l}\text { - Webpages, videos, image files, } \\
\text { PDFs, text blocks }\end{array}$ \\
\hline Tutorial structure & $\begin{array}{l}\text { - Based on author-defined } \\
\text { "chapters" }\end{array}$ & - Based on media-defined slides \\
\hline Reusability & $\begin{array}{l}\text { - Supports in-house copying } \\
\text { and templating } \\
\text { - Does not support external } \\
\text { copying }\end{array}$ & $\begin{array}{l}\text { - Supports in-house copying and } \\
\text { templating } \\
\text { - Does not support external } \\
\text { copying }\end{array}$ \\
\hline In-tutorial feedback & $\begin{array}{l}\text { - Can provide affirmative } \\
\text { feedback } \\
\text { - Can provide specific } \\
\text { corrective feedback }\end{array}$ & $\begin{array}{l}\text { - Can provide affirmative } \\
\text { feedback } \\
\text { - Can provide general corrective } \\
\text { feedback }\end{array}$ \\
\hline Tutorial scoring & $\begin{array}{l}\text { - Regular questions score last- } \\
\text { submitted responses } \\
\text { - Quiz questions score first- } \\
\text { submitted responses }\end{array}$ & $\begin{array}{l}\text { - All questions score first- } \\
\text { submitted responses }\end{array}$ \\
\hline Learner data & $\begin{array}{l}\text { - Individual email certificates } \\
\text { - Stored on local server }\end{array}$ & $\begin{array}{l}\text { - Automatic data aggregation } \\
\text { - Stored on vendor's server }\end{array}$ \\
\hline Accessibility & $\begin{array}{l}\text { - Single-page version is } \\
\text { compatible with some } \\
\text { accessibility needs }\end{array}$ & $\begin{array}{l}\text { - Learner view is 508-compliant } \\
\text { according to VPAT }\end{array}$ \\
\hline
\end{tabular}

TABLE 1. Summary of GotS and LibWizard key features.

Libraries that are considering adopting a platform for split-screen, live-web tutorials will find one of these options to better fit their needs, depending on the goals of their instruction program, their financial and human resources for administration and support, and their philosophies on questions like open source vs. proprietary tools. Local needs and priorities will guide libraries towards the platform characteristics that will be the most significant factors in their decisions. 
Further development appears likely for both platforms. The University of Arizona Libraries and Springshare are both receptive to feedback from their platform users. Both maintain lists of desirable fixes and enhancements. And now that there are alternative products for the split-screen model, perhaps each platform will learn from the other. One platform's developers may identify enhancements that are present in the other platform, or hear from users about what they think their platform of choice is missing. It is also conceivable that similar products will be released, given the size of the market across higher education and the rising commercial value of student data.

Another hope for the future is the growth of the user community. Adoption and use of these platforms may become more widespread as libraries continue to seek effective ways to flip classroom instruction and extend online instruction. The research so far on the effectiveness of this model of tutorial indicates it is an effective mode of online instruction. Further research that substantiates this finding would reinforce the incentives to adopt this approach to tutorial creation. There is also a need for investigations on the production side, especially studies that can establish a clearer picture of the time-costs of administration, support, design, development, and updating.

Adoption by more librarians and the production of more split-screen tutorials would generate greater conversation about pedagogical practice, sustainable administration, and usability. It could also add to the number of high-quality tutorials that inspire others and stimulate more thinking about how to support reusability. The technology, while still emerging through product iterations, is in place. Now, librarians need to get the most out of it. 


\section{References}

Bracke, Paul J., and Ruth Dickstein. 2002. "Web Tutorials and Scalable Instruction: Testing the Waters.” Reference Services Review 30 (4): 330-37. doi:10.1108/00907320210451321. caosborne89, simpsonw, michaelhagedon, and ottenhoff. 2016. "Guide-on-the-

Side/README.md." GitHub. July 27. https://github.com/ualibraries/Guide-on-the-Side. DeFrain, Erica. 2013. “Evaluating Information.” June. http://library.uvm.edu/guide_on_the_side/tutorial/evaluating-information.

Free, Michael. 2013. "Hagedon and Sult win 2013 ACRL IS Innovation Award." American Library Association. March 25. http://www.ala.org/news/pressreleases/2013/03/hagedon-and-sult-win-2013-acrl-innovation-award.

Hansen, Robert, and Jeremiah Grossman. 2008. “Clickjacking.” SecTheory: Internet Security. September 12. http://www.sectheory.com/clickjacking.htm.

Kaletski, Grace. 2016. "LibWizard.” The Charleston Adviser, July 1.

Mery, Yvonne, Erica DeFrain, Elizabeth Kline, and Leslie Sult. 2014. "Evaluating the Effectiveness of Tools for Online Database Instruction." Communications in Information Literacy 8 (1): 70-81.

Mery, Yvonne, Leslie Sult, and Erica DeFrain. 2013. “A New Spin on an Old Classic: Effective Online Database Instruction." presented at LOEX 2013, Nashville, TN. http://www.loexconference.org/2013/sessions.html\#mery.

mhagedon. 2016. "Guide on the Side 1.0-beta4 Released." http://code.library.arizona.edu/guideside-1-beta4-released. 
Mikkelsen, Susan, and Elizabeth McMunn-Tetangco. 2014. "Guide on the Side: Testing the Tool and the Tutorials." Internet Reference Services Quarterly 19 (3-4): 271-82. doi:10.1080/10875301.2014.948252.

Pawlek, Sarah. 2016a. "Introducing LibWizard." Springshare. February 3. http://blog.springshare.com/2016/02/03/introducing-libwizard/.

—.2016b. "LibWizard 1.7.6 Now Live!” Springshare. October 19. http://blog.springshare.com/2016/10/19/libwizard-1-7-6-now-live/.

_. n.d. "Voluntary Product Evaluation Template (VPAT)."

http://ask.springshare.com/loader.php?fid=37571\&type $=0 \& k e y=6 \mathrm{~b} 58 \mathrm{c} 384 \mathrm{c} 3280 \mathrm{ce} 034 \mathrm{fd}$ 9ac65f54e718.

Race, Phil. 2006. The Lecturer's Toolkit: A Practical Guide to Learning, Teaching and Assessment. 3 edition. London: Routledge.

simpsonw, and michaelhagedon. 2008. "Release Notes." GitHub. November 11. https://github.com/ualibraries/Guide-on-the-Side.

Snediker, Kathy. 2015. “Search for History Books.” November.

\section{http://GotS.tcl.sc.edu/tutorial/history-books.}

Stonebraker, Ilana. 2015. “Specialized Tutorials for Specialized Resources: Using Interactive Guide on the Side Tutorials for Special Libraries Reference and Instruction.” Public Services Quarterly 11 (3): 232-36. doi:10.1080/15228959.2015.1060145.

Stonebraker, Ilana, M. Brooke Robertshaw, and Jennifer D. Moss. 2016. "Student See Versus Student Do: A Comparative Study of Two Online Tutorials.” TechTrends 60 (2): 176-82. doi:10.1007/s11528-016-0026-7. 
Sult, Leslie, Yvonne Mery, Rebecca Blakiston, and Elizabeth Kline. 2013. “A New Approach to Online Database Instruction: Developing the Guide on the Side." Reference Services Review 41 (1): 125-33. doi:http://dx.doi.org.lscproxy.libraries.vsc.edu/10.1108/00907321311300947.

United States Access Board. 2000. "Section 508 Standards for Electronic and Information Technology." United States Access Board. December 21. https://www.accessboard.gov/guidelines-and-standards/communications-and-it/about-the-section-508standards/section-508-standards.

\footnotetext{
${ }^{1}$ Frames are boxes into which the browser screen is divided in order to organize the display. They should not be confused with iframes, which are boxes that can be individually embedded in the browser screen.

${ }^{2}$ This is often a security-based decision to block clickjacking, a hostile practice where a website tricks a user into clicking its links or submitting data by overlaying the real site with a frame displaying a page from a trusted site (Hansen and Grossman 2008).

${ }^{3}$ Using a coding editor, which would be more time-intensive than using the current version's text editor. The estimated time for development does not include design, validation, and redesign.

${ }^{4}$ Accessible via the online guide for one of the FWIL courses:

http://researchguides.uvm.edu/english1/tutorials.

${ }^{5}$ Online documentation is available only to logged-in LibApps account holders.
} 
${ }^{6}$ A "Regular" LibWizard user cannot access another user's tutorials or their tutorial data through the admin-side listing of tutorials, but they can access another user's text items and questions via the Question Bank.

${ }^{7}$ The provision of this information is itself one of the standards in Section 508, $\$ 1194.41$ (b):

"End-users shall have access to a description of the accessibility and compatibility features of

products in alternate formats or alternate methods upon request, at no additional charge." (United States Access Board 2000) 